\title{
A Comparative Study on Efficiency of Classification Techniques with Zone Level Gabor Features towards Handwritten Telugu Character Recognition
}

\author{
N. Shobha Rani \\ Maharaja Research \\ Foundation \\ University of Mysore \\ Maharaja institute of technology, \\ Mysore
}

\author{
Vasudev T. \\ Maharaja Research Founda \\ University of Mysore \\ Maharaja institute of technology, \\ Mysore
}

\begin{abstract}
Achieving high accuracies in recognition of handwritten text is a challenging research problem and never exhausting. The factors that instill challenges in handwritten character recognition include high degree of variability in writing, script type and the type of documents etc. In this paper, we focus on recognition of handwritten Telugu text commonly found in document images. The character set include all the vowels, consonants and single level vowel consonant clusters chosen in accordance with the commonly used terminology employed in composition pre-printed documents such as admission forms. In this paper, an algorithm is devised that performs zone-based feature extraction of the segmented character images. In the proposed work, the Gabor features are extracted from the character image at zone level and its efficiency is evaluated individually on two different zone representations and entire image at various scales and orientations. The classification and recognition performance is analyzed using nearest neighbor classifier, Naïve Bayesian, multi-class SVM and probabilistic neural networks classifiers. The efficiency of the classifiers are also tested with statistical, Histogram of Gradients and $\mathrm{Hu}$ moments' feature extraction methods and the best accuracy of the system is found to be $84.8 \%$ for Gabor features with zone representation 2 and with multi-class SVM classifier.
\end{abstract}

\section{Keywords}

Handwritten Telugu text, Character recognition, Gabor feature, Multi-class SVM, Naïve Bayesian classifier, nearest neighbor.

\section{INTRODUCTION}

Character recognition systems in today's generation have significant usages in various organizations to improvise the efficiency of the work flow. The recognition of text by optical technology like Optical Character Recognition (OCR) is a vast problem domain in the area of pattern recognition and computer vision. The major categories of documents include printed and handwritten text. The printed text is associated with two important attributes; font style and script from which it is composed. The handwritten text is variant with respect to every individual who author it. The recognition system that necessitates the recognition of printed text is not applicable for recognition of handwritten text. More over the software requirements of OCR varies from one type of script to another. The complexity involved in handling a wider problem domain with a generic OCR can be minimized by focusing on to recognition of only specific script and document, text type (printed or handwritten), font style, specific document type and places of document usage etc.
This also leads to reduced computational complexity and an improved accuracy in recognition. This may subsequently result in optimal speed in work flow of various organizations where the technology usage is of major concern.

The recognition of text in document image is performed through a sequential model comprising of pre-processing, object detection, feature extraction, classification, recognition and post-processing. The output of each stage is very vital in attaining the overall accuracy of the recognition by OCR. The feature extraction is one of the crucial stages which require to compute some features from detected objects that can provide the unique recognition of that object in an image. The objective of present work lies in devising an efficient processing model for feature extraction and classification of Telugu handwritten characters.

The conventional feature extraction methods include statistical, structural and global transformations. The statistical features [1] are characterized by zoning, projections profiles, crossings and distances which include density, direction features and projection histograms etc. Some of the structural features [2] of the image comprises aspect ratio, percentage of black pixels above the horizontal half point, percentage of black pixels to the right of vertical half point, number of strokes, average distance from image center, reflections from $\mathrm{Y}$-axis and reflection of image from $\mathrm{X}$-axis etc. Finally, the global features [3] of the image, which can be computed either in the spatial domain or frequency domain. The Fourier, discrete cosine transforms and other transformations are applied on the character image prior to feature computation. The statistical and structural features are the best supported for the recognition of printed text rather handwritten text due to variability in features of same character. These features returns the different set of features leading to misrecognition. Also, the high degree of variability from printed to handwritten text of same character creates the inconsistency between OCR for printed documents and handwritten documents.

On the other hand, the feature computation of character images can be performed either on binary images or grayscale images [4]. The computation of features from binary images may result in good accuracy and reduced complexity in case of printed documents and may produce inconsistent results for handwritten documents due to handwriting variability and fixed nature of binary images. The recognition of handwritten characters through features computed from its gray scale counter parts may lead to appreciable outcomes since the transformations applied are global in nature and also robust for the same character at various degrees, orientations and 
scales [5]. The feature computation on gray scale images employed at zone level [6] may lead to more discriminable features, as some of the zones carry only distinct information of a character. Especially in South Indian languages like Telugu, Kannada most of the characters have similar resemblance and performing the feature computation at zone level increases the scope of accurate recognition.

The recognition of handwritten text in this work is performed through zone level feature extraction from each character image. The local features [7] of few zones are beneficial when there is rough segmentation of character image and also the instances of two or more characters of similar resemblance. Thus zone level features are efficient for handwritten character recognition and in the present work Gabor features are extracted from each zone and further these features are directed for classification.

\section{LITERATURE SURVEY}

Numerous attempts were reported in the literature for the feature extraction and classification and some of important and relevant works are discussed.

George et. al [8] has proposed a feature extraction algorithm for recognition of handwritten characters using bi-orthogonal wavelets and employed curvature function and contours for identification of character using LVQ algorithms. The experimentation is performed on NIST datasets of handwritten numerals and achieved an accuracy of around $90 \%$, the number of classes in numerals is only 10 where as for the South Indian languages it is more than 100 including vowels, consonants, single level vowel consonant clusters. Pritpal et. al[9], Sumedha et. al [10], Gita et. al. [11] and Sandeep et. al[12] has proposed a zone based hybrid approach for recognition of Gurumukhi script, handwritten Gurumukhi numerals and handwritten English characters using distance features computed from centroid of character and nearest neighbor, SVM and neural network classifier for classification, the accuracy of the individual experimentations is almost $95 \%, 88 \%, 99 \%$ and $83.84 \%$ respectively. Raju et. al [13] had analyzed the performance of 2D-FFT features and SVM classifier on printed Telugu text documents and accuracy attained is about $71 \%$. Vikram et. al. [14] has employed the multi layer perceptrons and analyzed the performance of recognition using Hidden Markov Models and Artificial neural network models, the accuracy is found to be 85\%. Ram et. al.[15] had proposed a comparision based approach for recognition of printed Telugu script of varying fonts and sizes. The algorithm performs zone based recognition. A database is maintained for the comparison purposes and these kinds of methods are not consistent enough for huge data samples and handwritten script due to very low discrimination range from one character to another character. Vijay et. al [16] had analyzed the performance of handwritten Telugu script using multi layer perceptron model and had obtained an accuracy of $81 \%$. Samit et. al [17] had proposed an approach for recognition of handwritten Telugu script using trie[18] data structure The algorithm has been tested only on 43 different basic characters and requires much computational burdens when the dataset size grows big. Dhandra et. al [19] had analyzed the peformance of probabilistic neural networks on recognition of numerals in Kannada, Telugu and Devanagari and obtained an accuracy of about $95 \%$. Narahari et. al [20] had analyzed the performance of decision tree classifier using SEE5 algorithm[21] on the Telugu characters extracted from palm leaves with an accuracy of around 93\%. Rasagna et. al [22] had analyzed the performance of HOG features and SVM classifier for recognition of Telugu printed characters of 15 different fonts and 359 different classes for which an accuracy of about $95 \%$ is attained. Nusaibath et. al.[23], Xeuewen[24], Liu et. al. [25], Xeuewen[26] and Jin et. al[27] had compared the performance of Gabor features for recognition and obtained an average accuracy of more than $90 \%$.

To the best of our knowledge, the review in literature, it is observed that most of the experimentations for recognition of characters are analyzed either on binary images or gray scale images. The recognition based on binary images is efficient for printed character recognition, where as for handwritten character analysis manipulations are performed on gray scale images of characters. The statistical features, zoning and geometrical features are suitable and consistent enough for classification of printed text. The Gabor features [28], wavelet features [29], moments [30], gradients [31] and template matching [32] techniques are extensively employed for classification and recognition of handwritten character images. Investigations in the area of printed Telugu characters are more reliable and consistent with variety of font styles and sizes with an overall accuracy of around $98 \%$. Also, it is observed that, the experimentations on South Indian scripts like Telugu can be still extended due to its reduced discrimination range from class of characters to the other especially in case of handwritten text. Therefore, the focus of the present work is to analyze the recognition rate with various classifiers by employing Gabor features through which an enhanced recognition rate can be achieved for handwritten Telugu characters.

\section{PROPOSED METHODOLOGY}

The accurate recognition of characters is accomplished only when the features rendered during the feature extraction stage are robust and distinctive for each of the character. In this work, we propose a processing model for feature computation and classification of Telugu handwritten characters. The block diagram of proposed work is presented in fig. 1 .

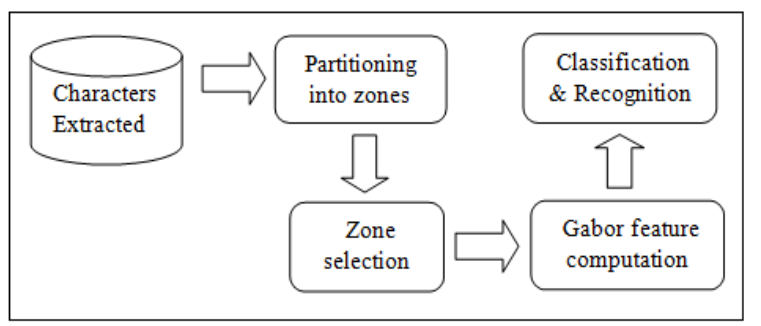

Fig. 1 Proposed model of feature computation and classification

Invocation of proposed algorithm assumes the input as the database of gray scale character images segmented from the document image. Each character image is resized to $39 \times 39$ and subjected for partitioning into zones. Performing the resizing/normalization prior to feature computation maintains the uniformity in behavior of algorithm. This may produce more discriminable features from character to another. As the handwritten characters are drafted with more flexibility and freedom compared to printed text, there may be two or more characters visualizing the same but scales are different and resulting into an interpretation as different character due to its scale. Therefore performing the resizing of character image prior to feature computation is a significant task. The partitioning of character image into zones and zones selection is described in the subsequent section 2.1. 


\subsection{Zone partitioning and zone selection}

The objective of partitioning the character image into zones is to maximize the distinct features of characters with similar resemblance and slight changes. The character set in Telugu script shares common set of features like curvature, concavity and convexity in almost all the characters with very slight differences, thus leading to redundant features for two or more different characters. By focusing the feature computation on specific zones or parts of the character image, it is possible to achieve required discrimination among the characters. The proposed model considers two different overlapping zone representations from which zones are selected for feature computations. The two zone representation models and their corresponding character image representations are depicted in fig. 2(a), 2(b), 2(c) and 2(d).

\begin{tabular}{|c|}
\hline Zone 1 \\
\hline Zone 2 \\
\hline Zone 3 \\
\hline
\end{tabular}

Fig. 2(a) Zone representation 1

\begin{tabular}{|l|l|l|}
\hline Zone 1 & Zone 2 & Zone 3 \\
\hline Zone 4 & Zone 5 & Zone 6 \\
\hline Zone 7 & Zone 8 & Zone 9 \\
\hline
\end{tabular}

Fig. 2(b) Zone representation 2

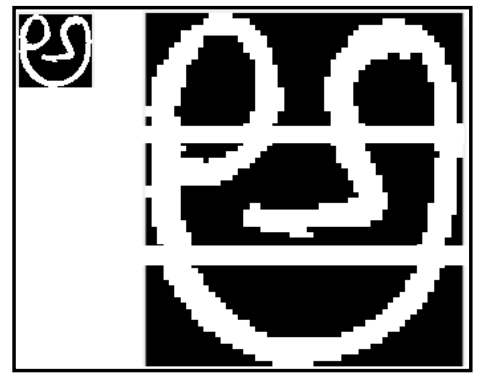

Fig. 2(c) Zone Representation1 of character image

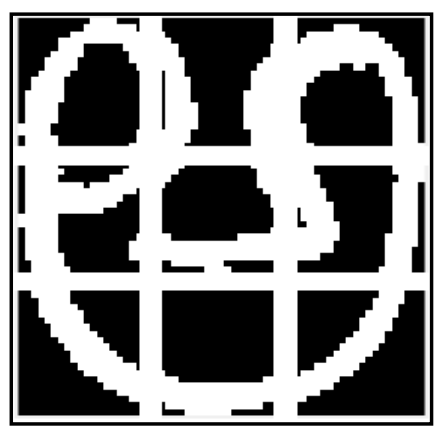

Fig. 2(d) Representation2 of character image

Telugu is a South Indian script consisting of 16 vowels, 36 consonants, single level and multi level vowel consonant clusters all generating together more than 550 distinct characters. The vowel consonant clusters are generated by the combination of one or more vowels, consonants and modifiers for vowels and consonants. As a result large number of character combinations, it introduces complexity as well as ambiguities in recognition of the character. The major complexity of recognition in Telugu script is characters exhibit similar resemblance in its topological and spatial characteristics like width strokes etc, leads to misrecognition. However as each character has its unique name and possess some degree of dissimilarities from the other, leading to a scope for better recognition. This small degree of dissimilarities or unique topological characteristics of character can be attained only when we decompose the character into zones. As few of the zones possess some unique characteristics describing the meaning of character and thus increases the chance of achieving good recognition results.

In the proposed method, we have chosen zone 1 in the first zone representation or zone 1 , zone 2 and zone 3 in zone representation 2. The second zone representation includes zone 2 , zone 3 , zone 4 , zone 6 , zone 8 and zone 9 as shown in second zone representation as these zones describe distinct characteristics of characters. The zones selected from two representations for feature computations are as shown in figure 3(a) and 3(b).

\begin{tabular}{|l|l|l|}
\hline Zone 1 & Zone 2 & Zone 3 \\
\hline Zone 4 & Zone 5 & Zone 6 \\
\hline Zone 7 & Zone 8 & Zone 9 \\
\hline
\end{tabular}

(a)

\begin{tabular}{|l|l|l|}
\hline Zone 1 & Zone 2 & Zone 3 \\
\hline Zone 4 & Zone 5 & Zone 6 \\
\hline Zone 7 & Zone 8 & Zone 9 \\
\hline
\end{tabular}

(b)

Fig. 3 Zones selected from representation $1 \& 2$

The feature extraction from the selected zones of two representations and for the entire character image is performed separately in the proposed methodology to facilitate the comparison of recognition accuracies in all the three cases. The Gabor feature extraction technique is employed on the gray scale versions of character images, the sub section 2.2 describes the feature extraction stage.

\subsection{Gabor feature computation}

The major problem in Telugu handwritten character recognition is similar geometrical properties in most of the characters defining the script, presence of confusing character pairs, common concavity features shared by characters in more than one zone, barriers of handwriting. The Gabor wavelet features [24] are employed in the proposed method, since it consists of data maintained at various frequencies and orientations making it suitable for extraction of discriminant features among characters. Gabor filters are directly related to 
Gabor wavelets and is a 2d-Gaussian kernel function, generally used for perception of high frequency components in the image leading to filter out only gradient details like textual components in the character image. The filter is defined of real and imaginary components forming together the complex number representing various orthogonal directions. The corresponding complex numbers along with its real and imaginary components are as given in equation (1), (2) and (3) respectively.

Complex number.,

$g(x, y ; \lambda, \theta, \psi, \sigma, \gamma)=\exp \left(-\frac{x^{\prime 2}+\gamma^{2} y^{\prime 2}}{2 \sigma^{2}}\right) \exp \left(i\left(2 \pi \frac{x^{\prime}}{\lambda}+\psi\right)\right)$

Real part.,

$g(x, y ; \lambda, \theta, \psi, \sigma, \gamma)=\exp \left(-\frac{x^{\prime 2}+\gamma^{2} y^{\prime 2}}{2 \sigma^{2}}\right) \cos \left(2 \pi \frac{x^{\prime}}{\lambda}+\psi\right)(2)$

Imaginary part.,

$g(x, y ; \lambda, \theta, \psi, \sigma, \gamma)=\exp \left(-\frac{x^{\prime 2}+\gamma^{2} y^{\prime 2}}{2 \sigma^{2}}\right) \sin \left(2 \pi \frac{x^{\prime}}{\lambda}+\psi\right)(3)$

where $x^{\prime}=x \cos \theta+y \sin \theta$ and

$y^{\prime}=-x \sin \theta+y \cos \theta$

$\lambda$ and $\theta$ are wave length and orientation of normal to parallel stripes of Gabor function. $\psi$ is phase offset, $\sigma$ is standard deviation and $\gamma$ is aspect ratio.

In the proposed method, the Gabor filter bank [33] is created with $2 \mathrm{~d}$ filter dimensions as 39x 39 and defined with 5 scales and 8 different rotations. The response from Gabor filter bank is a cell array of dimension $5 \times 8$ and each element is a matrix of size $39 \times 39$. The details of Gabor filter bank is as depicted in fig. 4 and fig. 5.

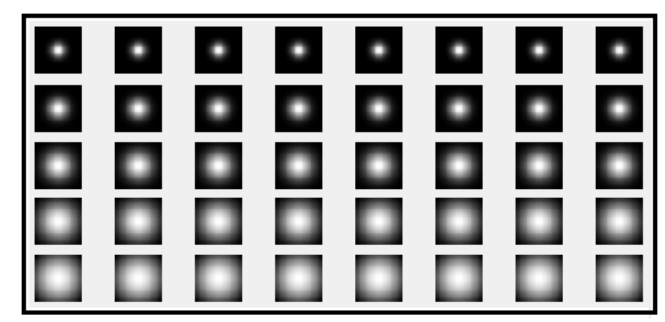

Fig. 4 Magnitudes of Gabor filter bank

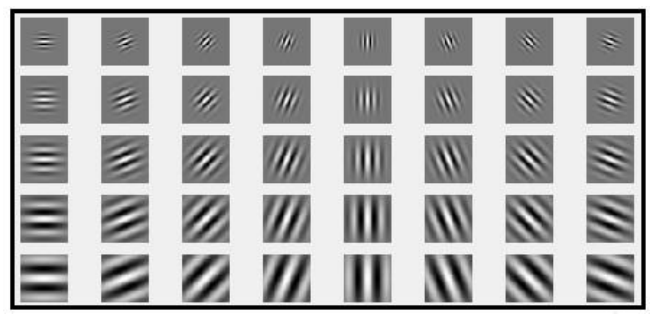

Fig. 5 Real parts of Gabor filter bank

The response obtained from Gabor filter bank after convolving a character image with magnitudes and real parts of filter bank are filtered images at 5 different scales and 8 different orientations and the filtered images are shown in fig. 6 and fig. 7 for the character shown in fig.6-7(a).

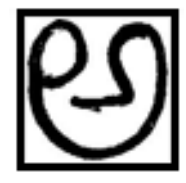

Fig. 6-7(a) A character image

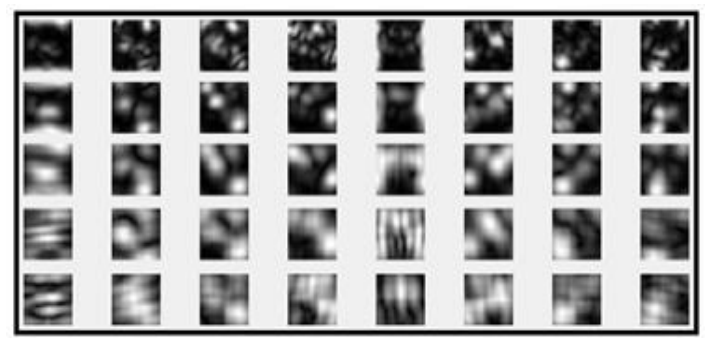

Fig. 6 Filtered images -Magnitudes

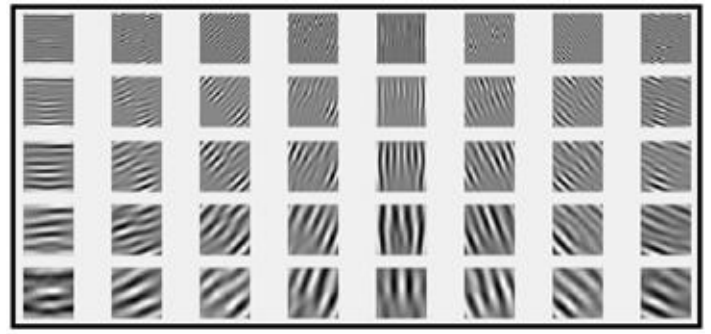

Fig. 7 Filtered images -Real parts

The real parts and magnitudes of the all the training images are computed separately for two different zone selections and entire image. The combined features of magnitude and real parts of each training set are stored as feature vectors. If $[I]_{Z 1},[I]_{Z 2}$ and $[I]_{E}$ represents the filtered images comprising of magnitudes and real parts of a character image $I$, then the Gabor feature vectors obtained from corresponding two zone selections and entire image are of dimensions $\left[f_{z 1}\right]_{2240 \times 1},\left[f_{z 2}\right]_{3920 \times 1}$ and $\left[f_{E}\right]_{7840 \times 1}$.

Further these features are employed for classification of characters.

\subsubsection{Training features generation}

Consider $I_{1}, I_{2}, I_{3} \ldots I_{n}$ as the instances of character images employed for training and $f_{1}, f_{2}, f_{3} \ldots f_{l}$ indicates the features of each instance, then the training vector $\tau$ is given by equation (4).

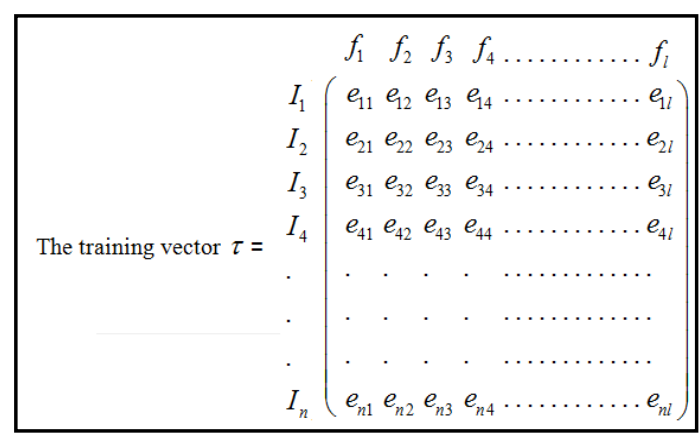

Equation (4) 
where, $e_{i j}$ corresponds to a feature $f_{j}$ of image $I_{i}$ and $i=1,2,3 \ldots n, j=1,2,3 \ldots l$.

Similarly, the test vector is also composed for the test set for validation with the tra0ining vector $\tau$. The target labels vector $\ell_{n \times 1}$ corresponding to each training instance is also created. The target label represents the class to which the training instance belongs to. The vector representation of target labels $\ell_{n \times 1}$ is given by equation (5).

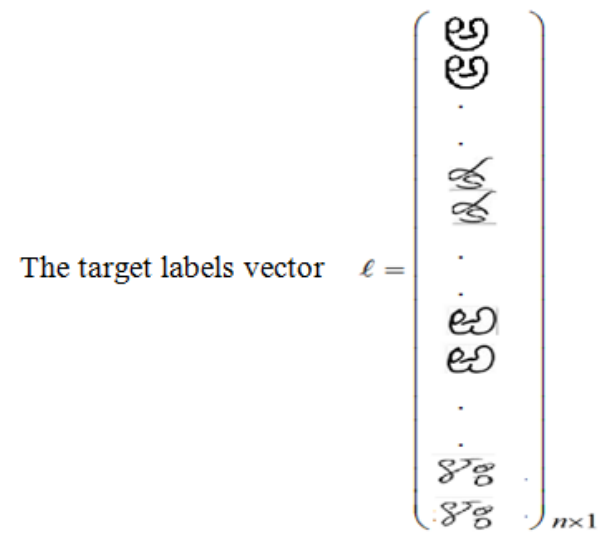

The repeated labels indicate that, the first training instance belongs to class ' $\mathrm{A}$ ' and second also belongs to the same class. The subsequent section describes the classification of features computed to their respective classes.

\subsection{Classification of features}

The classification of the features in the proposed method is performed by employing various classifiers like nearest neighbor classifier, Multi-class SVM, Naïve Bayesian and probabilistic neural networks to facilitate the comparative study of recognition outcomes with respect to various classifiers as well as different zone selections.

\subsubsection{Nearest neighbor classification}

The classification of a handwritten character to its target class is a non-trivial problem in character recognition systems. The nearest neighbor technique maps an input character to one of the target classes based on the measure of similarity between the test image and target images in the database. The similarity measure is a function of distance which includes the chamfer distance and Hausdorff matching for edge images, the Kullback-Leibler distance and the Earth Mover's Distance for probability distributions, and bipartite matching for sets of features etc are generally employed for pattern recognition problem [34]. The classification of the test character features is mapped to a target class based on the majority voting of the features. If $T e_{1}, T e_{2} \ldots T e_{n}$ represents the test samples and $f e_{1}, f e_{2}, f e_{3} \ldots f e_{n}$ indicates the feature vectors of test samples, then the test samples are classified to their respective classes $C_{1}, C_{2}, C_{3} \ldots C_{n}$ of training samples $\operatorname{Tr}_{1}, \operatorname{Tr}_{2}, \operatorname{Tr}_{3} \ldots T r_{n}$ corresponding to the voting of feature vectors $f r_{1}, f r_{2}, f r_{3} \ldots f r_{n}$. The voting of features between the test and training vectors are determined using the distance function as given by equation (6).

$$
D\left(T e_{n}, T r_{n}\right)=\left[\left(f e_{i}-f r_{i}\right)^{2}\right]^{1 / 2}
$$

The training and testing vectors for the nearest neighbor classifier requires the same number of features and also the number of instances in training vector should be equal to the number of classes available in the labels vector. The nearest neighbor classifier performs the multi modal classification and can provide the good accuracy when there is good discriminant range for various subsets of features. The computational complexity of the nearest neighbor classifier depends on the use of similarity measures or distance functions.

\subsubsection{Multi-class SVM classifier}

Support vector machines are originally designed for binary classification, however in real time for the problems of type optical character recognition several binary classifiers are combined to perform multi-class mapping [28]. Multi class SVM considers the multiple classes as one versus the rest according to the maximal output produced by the classification function. Consider a labeled training vector $T r_{1}, \operatorname{Tr}_{2}, \operatorname{Tr}_{3} \ldots T r_{n}$, the decision function is as given by equation (7) described by [35].

$\arg \max _{m} f_{m}(x)=\arg \max _{m}\left(W_{m}^{T} \varphi(x)+b_{m}\right)$

The $m^{\text {th }}$ binary classifier is trained using the data from the $m^{\text {th }}$ class as positive examples and the remaining $(k-1)$ classes as negative examples. During test, the class label is determined by the binary classifier that gives maximum output value.

\subsubsection{Nä̈ve Bayesian Classifier}

Naïve Bayesian classifiers [36] are probabilistic classifiers with assumptions of class conditional independence between the features. Let $f_{1}, f_{2}, f_{3} \ldots f_{n}$ represents ' $n$ ' features of a test sample $T e_{i}$ to be classified for which the prior probabilities assigned are $p\left(C_{k} \mid f_{1}, f_{2}, f_{3} \ldots f_{n}\right)$, where $C_{k}$ the number of classes and the posterior probability is decomposed as in equation (8).

$$
p\left(C_{k} \mid x\right)=\frac{p\left(C_{k}\right) p\left(x \mid C_{k}\right)}{p(x)}
$$

\subsubsection{Probabilistic Neural Networks}

Probabilistic Neural Network (PNN) is a feed forward neural network which performs kernel discriminant analysis through the input layer, pattern layer, summation layer and output layer [37]. The input layer represents the predictor variable and pattern layer corresponds to a single neuron for each of the input in the training set for which a weighted value is determined using the sigma function in the summation and the neuron with maximal probability score is considered as target variable or class. The probabilistic neural networks are insensitive towards the outliers and can exhibit robust classification outcomes. The architecture of PNN defined in the proposed system for two different zone selections as well as the entire character image are depicted in figure 8 , figure 9 and figure 10 respectively. 


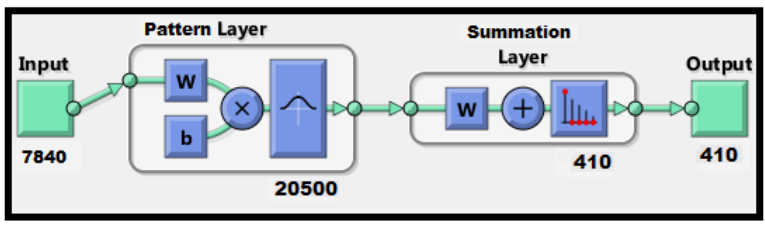

Fig. 8 Architecture of neural network for image

The probabilistic neural network in figure 8 is trained for recognition of a character which includes features of entire character image. The Gabor features of an entire image comprise a feature set of a total 7840 features for one character instance. Similarly, the training set dimensions is of $20500 \times 7840$ representing 20500 character images and its features.

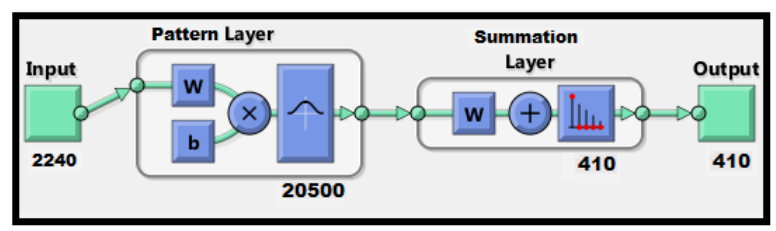

Fig. 9 Architecture of PNN for Zone representation 1

The probabilistic neural network in figure 9 is trained for recognition of a character which includes features of only zone 1 , zone 2 and zone 3 called as zone representation 1 . The Gabor features of zone representation 1 of character image comprise a feature set of a total 2240 features for one character instance. Similarly, the training set dimensions is of 20500x2240 representing 20500 character images and its features.

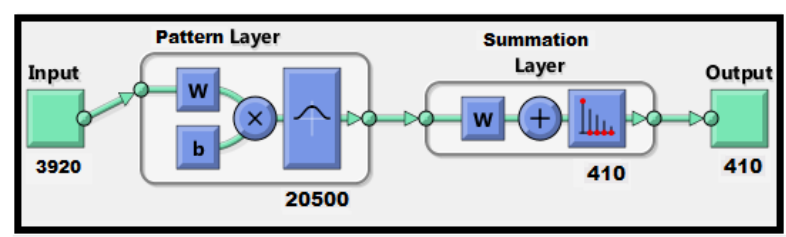

Fig. 10 Architecture of PNN for Zone representation 2

The probabilistic neural network in figure 10 is trained for recognition of a character which includes features of zone 1 , 2, 3, 6 and 9 of the character image. The Gabor features of an entire image comprise a feature set of a total 3920 features for one character instance. Similarly, the training set dimensions is of 20500x3920 representing 20500 character images and its features. The experimental analysis of the proposed system is presented in section 3 .

\section{EXPERIMENTAL ANALYSIS}

The experimentation in the proposed system is carried out with a synthetically generated datasets of Telugu handwritten characters. The dataset includes vowels, consonants, single level vowel-consonant clusters (Guninthalu) with around 50 references for each character composing the training set and 20 references is composed of test set. The dataset is generated by collecting the handwriting from around 90 different users and it eliminates all the infrequently used vowel consonant combinations like Nya, Jhah, Inya, Ksha, Ara etc. The entire dataset includes 410 distinct characters and classes for the same forming together around $410 \times 50$ characters in the training set and $410 \times 20$ characters in the test set which leads to 20500 and 8200 characters respectively. The Gabor features extracted from these datasets for two different zone representations and also for the entire character image is tested with nearest neighbor classifier, multi-class SVM, Naïve Bayesian and probabilistic neural network classifier.

If $T r_{1}, T r_{2}, T r_{3} \ldots T r_{n}$ and $T e_{1}, T e_{2}, T e_{3} \ldots T e_{n}$ represents the training and test samples, then the accuracy of classification is defined as the number of instances of the sequence $T e_{i} \in C_{k}$ where $C_{k}$ is the class to which $T e_{i}$ belongs to, $i=1,2,3 \ldots n$, $k=1,2,3 \ldots n$ and $i=k$.

Here $i=k$ indicates that $T e_{1} \in C_{1}, T e_{2} \in C_{2} \ldots T e_{n} \in C_{n}$. The accuracy is given by the equation (9).

$$
\text { Accuracy }=\frac{n_{c}}{\tau}
$$

where $n_{c}$ represents the number of test samples classified correctly by the classifier and $\tau$ is the total of characters in the test dataset.

The experimental evaluation has also been analyzed with the help standard performance measures [38] for testing classification accuracy. The performance measures such as sensitivity/recall rate, specificity, precision, F-measure and accuracy are employed for analyzing the performance of classification using Gabor features.

Let $N\left(T P^{+}\right), N\left(F N^{-}\right), N\left(T N^{-}\right)$and $N\left(F P^{+}\right)$represents the number of true positives, false negatives, true negatives and false positives respectively. The performance measures sensitivity, specificity, precision, F-measure and accuracy is defined by equation (10) through (14).

$$
\begin{aligned}
& \text { Sensitivity }=\frac{N\left(T P^{+}\right)}{N\left(T P^{+}\right)+N\left(F N^{-}\right)} \\
& \text {Specificity }=\frac{N\left(T N^{-}\right)}{N\left(T N^{-}\right)+N\left(F P^{+}\right)} \\
& \text {Precision }=\frac{N\left(T P^{+}\right)}{N\left(T P^{+}\right)+N\left(F P^{+}\right)}
\end{aligned}
$$

$$
F-\text { Measure }=2 \times \frac{\operatorname{Pr} \text { ecision } \times \text { Re call }}{\operatorname{Pr} \text { ecision }+\operatorname{Re} \text { call }}
$$

$$
\text { Accuracy }=\frac{N\left(T P^{+}\right)+N\left(T N^{-}\right)}{N\left(T P^{+}\right)+N\left(T N^{-}\right)+N\left(F P^{+}\right)+N\left(F N^{-}\right)}
$$

For experimentation, a total of 8200 handwritten test samples are employed in which 278 are noisy or cluttered character images whereas 7922 are clear and precise samples. In our analysis, we have considered $N\left(T P^{+}\right)$represents the noisy samples for which the class is correctly predicted, $N\left(F N^{-}\right)$ represents the noisy samples for which the class is incorrectly predicted, $N\left(F^{+}\right)$represents the non-noisy samples for which the class is incorrectly predicted and $N\left(T N^{-}\right)$ represents the non-noisy samples for which the class is correctly predicted. The results of experimentation statistics of 
zone representation 1, zone representation 2 and entire image for each of the classifier is as depicted in fig. 11 through fig. 16 respectively.

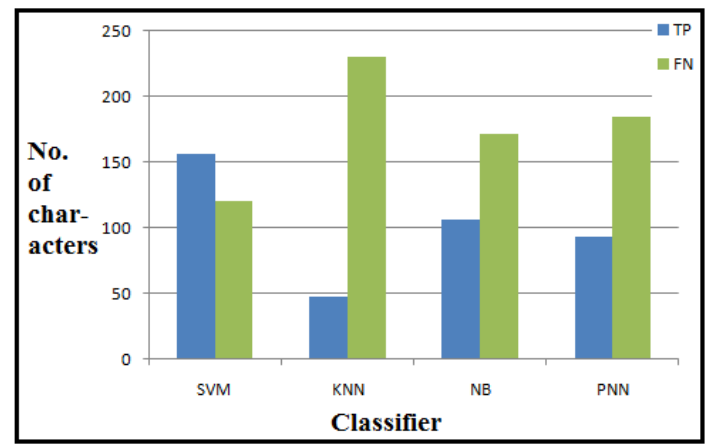

Fig. 11 Zone selections: $1,2 \& 3$

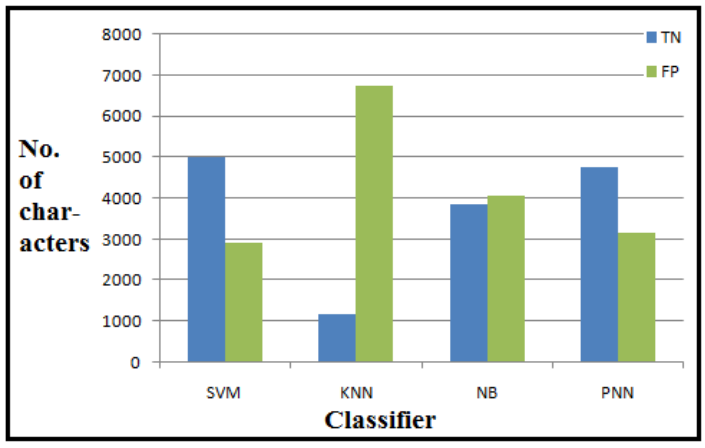

Fig. 12 Zone selections: 1, 2, \& 3

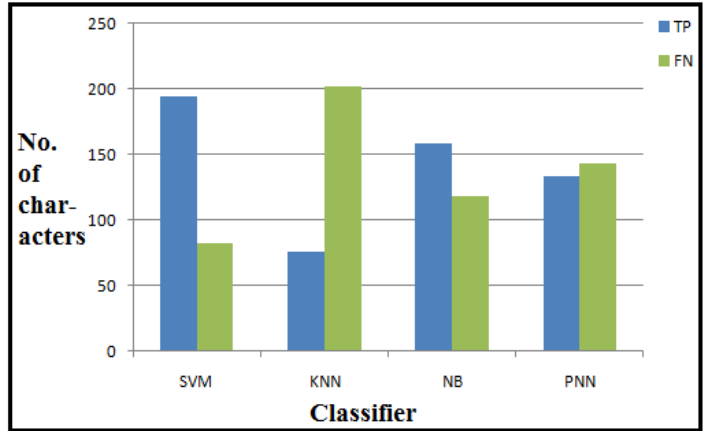

Fig. 13. Zone selections: 1, 2, 3 ,4, 6 \& 9

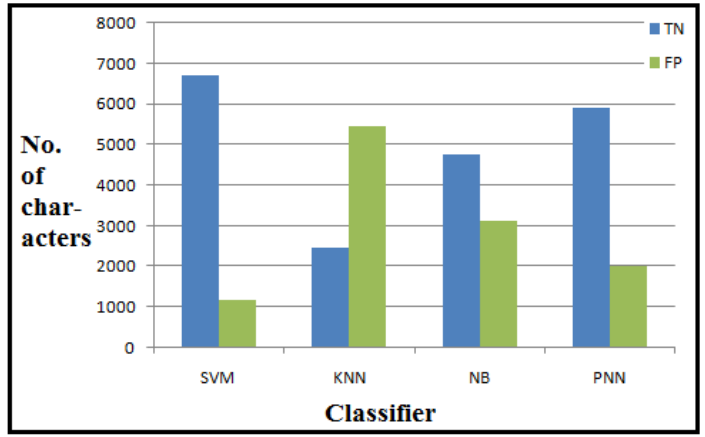

Fig. 14. Zone selections: 1, 2, 3, 4, $6 \& 9$

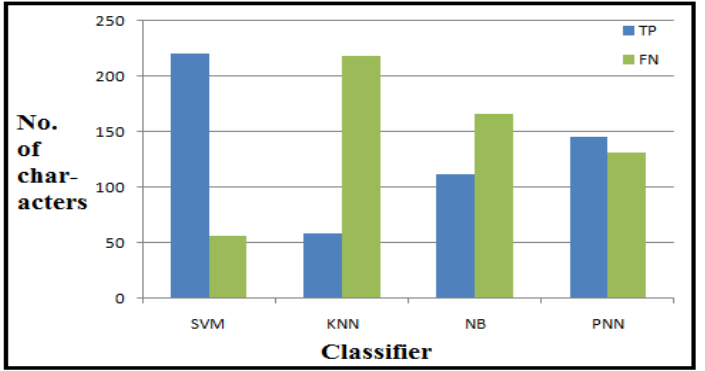

Fig. 15. Zone selections: Entire image

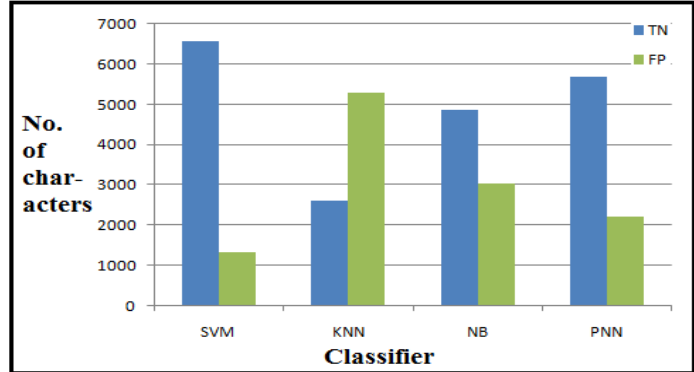

Fig. 16. Zone selections: Entire image

The efficiency of each classifier with reference to its two zone representations and entire character image has been enlisted with various performance measures for validating classifier with Gabor features is as listed in table 1.

Table1. Experimental evaluation- Performance of classifiers-Gabor features

\begin{tabular}{|c|c|c|c|}
\hline Classifier & Zone selection & $\begin{array}{l}\text { Sensit } \\
\text { ivity } \\
\% \\
\end{array}$ & $\begin{array}{l}\text { Specif } \\
\text { icity } \\
\% \\
\end{array}$ \\
\hline \multirow{3}{*}{$\begin{array}{c}\text { K-Nearest } \\
\text { neighbor }\end{array}$} & Zone 1,2 and 3 & 17.26 & 14.73 \\
\hline & Zone $2,3,4,6,8 \& 9$ & 27.33 & 31.04 \\
\hline & Entire image & 21.22 & 33.22 \\
\hline \multirow{3}{*}{$\begin{array}{c}\text { Naïve } \\
\text { Bayesian }\end{array}$} & Zone 1,2 and 3 & 38.12 & 48.67 \\
\hline & Zone $2,3,4,6,8 \& 9$ & 57.19 & 60.36 \\
\hline & Entire image & 40.28 & 61.61 \\
\hline \multirow{3}{*}{$\begin{array}{c}\text { Probabilistic } \\
\text { neural } \\
\text { networks }\end{array}$} & Zone 1,2 and 3 & 33.45 & 60.26 \\
\hline & Zone $2,3,4,6,8 \& 9$ & 48.2 & 74.52 \\
\hline & Entire image & 52.51 & 71.81 \\
\hline \multirow{3}{*}{$\begin{array}{l}\text { Support } \\
\text { vector } \\
\text { machines }\end{array}$} & Zone 1,2 and 3 & 56.47 & 63.21 \\
\hline & Zone $2,3,4,6,8 \& 9$ & 70.14 & 84.87 \\
\hline & Entire image & 79.49 & 83.14 \\
\hline
\end{tabular}

\begin{tabular}{|c|c|c|c|c|}
\hline \multirow{4}{*}{ Classifier } & Zone selection & $\begin{array}{c}\text { Precis } \\
\text { ion } \\
\text { \% }\end{array}$ & $\begin{array}{c}\text { F- } \\
\text { Mea } \\
\text { sure } \\
\text { \% }\end{array}$ & $\begin{array}{c}\text { Accur } \\
\text { acy } \\
\text { \% }\end{array}$ \\
\hline \multirow{3}{*}{$\begin{array}{c}\text { K-Nearest } \\
\text { neighbor }\end{array}$} & Zone 1, 2 and 3 & 0.7 & 1.34 & 14.81 \\
\cline { 2 - 5 } & Zone 2,3,4,6,8 \& 9 & 1.37 & 2.60 & 30.91 \\
\cline { 2 - 5 } & Entire image & 1.1 & 2.09 & 32.81 \\
\hline \multirow{2}{*}{$\begin{array}{c}\text { Naïve } \\
\text { Bayesian }\end{array}$} & Zone 1, 2 and 3 & 2.54 & 4.76 & 48.31 \\
\cline { 2 - 5 } & Zone 2,3,4,6,8 \& 9 & 4.81 & 8.87 & 60.25 \\
\hline
\end{tabular}




\begin{tabular}{|c|c|c|c|c|}
\hline & Entire image & 3.55 & 6.52 & 60.89 \\
\hline \multirow{3}{*}{$\begin{array}{c}\text { Probabilisti } \\
\mathrm{c} \text { neural } \\
\text { networks }\end{array}$} & Zone 1,2 and 3 & 2.86 & 5.26 & 59.35 \\
\hline & Zone $2,3,4,6,8 \& 9$ & 6.22 & $\begin{array}{c}11.0 \\
1\end{array}$ & 73.63 \\
\hline & Entire image & 6.13 & $\begin{array}{c}10.9 \\
7\end{array}$ & 71.15 \\
\hline \multirow{3}{*}{$\begin{array}{l}\text { Support } \\
\text { vector } \\
\text { machines }\end{array}$} & Zone 1,2 and 3 & 5.11 & 9.37 & 62.98 \\
\hline & Zone $2,3,4,6,8 \& 9$ & 13.99 & $\begin{array}{c}23.3 \\
2\end{array}$ & 84.37 \\
\hline & Entire image & 14.2 & $\begin{array}{c}24.0 \\
9\end{array}$ & 83.02 \\
\hline
\end{tabular}

The results of classification tested with various classifiers with respect to different zone selections are described below. The Receiver Operating Characteristic (ROC) curve representing the PNN classifier receiving rate is as shown in fig. 17.

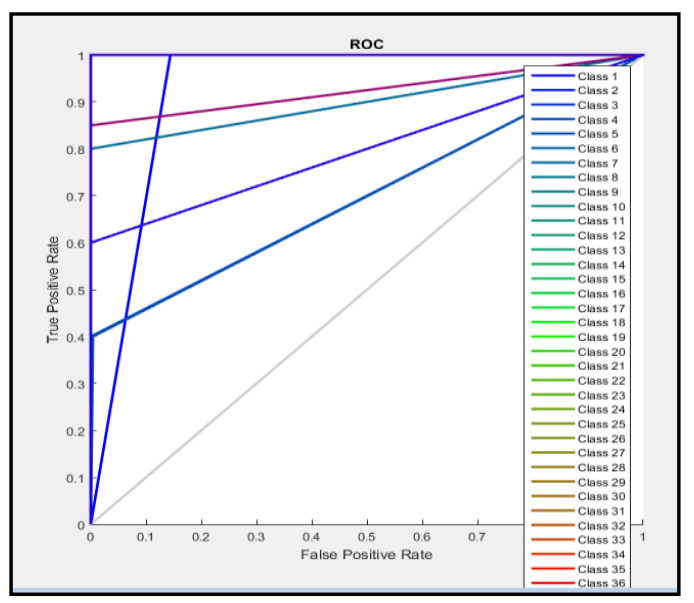

Fig. 17 ROC-Probabilistic neural networks

The experimentation is also conducted with the statistical features [1], Histogram of Gradients (HoG) features[39] and $\mathrm{Hu}$ moments features[40] and the results of the experimentation with various classifiers is depicted in the table 2, table 3 and table 4 .

Table 2. Experimental evaluation- Performance of classifiers- Statistical features

\begin{tabular}{|c|l|r|r|}
\hline \multirow{4}{*}{ Classifier } & Zone selection & \multicolumn{1}{|l}{$\begin{array}{l}\text { Sensitiv } \\
\text {-ity }\end{array}$} & \multicolumn{1}{|l}{$\begin{array}{l}\text { Specif } \\
\text { icity } \\
\text { \% }\end{array}$} \\
\hline \multirow{4}{*}{$\begin{array}{c}\text { Nearest } \\
\text { neighbor }\end{array}$} & Zone 1, 2 and 3 & 6.11 & 1.52 \\
\cline { 2 - 4 } & Zone 2,3,4, 6,8 \& 9 & 11.8 & 13.7 \\
\cline { 2 - 4 } & Entire image & 6.47 & 5.42 \\
\hline \multirow{4}{*}{$\begin{array}{c}\text { Naïve Bayes } \\
\text { Probabilistic } \\
\text { neural } \\
\text { networks }\end{array}$} & Zone 1, 2 and 3 & 10.43 & 7.54 \\
\cline { 2 - 4 } & Zone 2,3,4, 6,8 \& 9 & 19.7 & 15.3 \\
\cline { 2 - 4 } & Zone 1, 2 and 3 & 13.3 & 6.55 \\
\cline { 2 - 4 } & Zntire image $2,3,4,6,8$ \& 9 & 32.01 & 11.15 \\
\hline \multirow{3}{*}{$\begin{array}{c}\text { Support vector } \\
\text { machines }\end{array}$} & Zone 1, 2 and 3 & 18.7 & 12.33 \\
\cline { 2 - 4 } & Zone 2,3,4, 6,8 \& 9 & 35.9 & 14.73 \\
\cline { 2 - 4 } & Entire image & 25.8 & 13.74 \\
\hline
\end{tabular}

\begin{tabular}{|c|l|l|l|r|}
\hline \multirow{4}{*}{ Classifier } & Zone selection & $\begin{array}{l}\text { Pre } \\
\text { cisi } \\
\text { on } \\
\text { \% }\end{array}$ & $\begin{array}{l}\text { F- } \\
\text { Meas } \\
\text { ure } \\
\text { \% }\end{array}$ & $\begin{array}{l}\text { Accura } \\
\text { cy } \\
\text { \% }\end{array}$ \\
\hline \multirow{4}{*}{$\begin{array}{c}\text { Nearest } \\
\text { neighbor }\end{array}$} & Zone 1, 2 and 3 & 0.21 & 0.40 & 1.68 \\
\cline { 2 - 5 } & Zone 2,3,4, 6,8 \& 9 & 0.47 & 0.90 & 13.7 \\
\hline \multirow{4}{*}{$\begin{array}{c}\text { Naïve } \\
\text { Bayes }\end{array}$} & Zntire image & 0.23 & 0.44 & 5.46 \\
\cline { 2 - 5 } & Zone 1, 2 and 3 & 0.39 & 0.75 & 7.64 \\
\cline { 2 - 5 } & Zntire image & 0.49 & 0.94 & 15.5 \\
\hline \multirow{3}{*}{$\begin{array}{c}\text { Probabilist } \\
\text { ic neural } \\
\text { networks }\end{array}$} & Zone 1, 2 and 3 & 0.81 & 1.55 & 6.78 \\
\cline { 2 - 5 } & Zone 2,3,4, 6,8 \& 9 & 1.47 & 2.81 & 25.1 \\
\hline \multirow{3}{*}{$\begin{array}{c}\text { Support } \\
\text { vector } \\
\text { machines }\end{array}$} & Zntire image & 0.74 & 1.42 & 12.54 \\
\cline { 2 - 5 } & Zone 1, 2 and 3 & 0.69 & 1.32 & 14.8 \\
\cline { 2 - 5 } & Zntire image $2,3,4,6,8 \& 9$ & 1.93 & 3.65 & 37.2 \\
\hline \multirow{2}{*}{} & & 1.04 & 1.99 & 14.15 \\
\hline
\end{tabular}

Table 3. Experimental evaluation- Performance of classifiers- HoG features

\begin{tabular}{|c|c|c|c|}
\hline Classifier & Zone selection & $\begin{array}{l}\text { Sensiti } \\
\text { vity } \\
\% \\
\end{array}$ & $\begin{array}{l}\text { Specifici } \\
\text { ty } \\
\% \\
\end{array}$ \\
\hline \multirow{3}{*}{$\begin{array}{c}\text { Nearest } \\
\text { neighbor }\end{array}$} & Zone 1,2 and 3 & 3.23 & 3.2 \\
\hline & Zone $2,3,4,6,8 \& 9$ & 11.5 & 15.9 \\
\hline & Entire image & 8.27 & 4.59 \\
\hline \multirow{3}{*}{$\begin{array}{l}\text { Naïve } \\
\text { Bayes }\end{array}$} & Zone 1,2 and 3 & 6.47 & 3.68 \\
\hline & Zone $2,3,4,6,8 \& 9$ & 20.8 & 18.8 \\
\hline & Entire image & 12.23 & 7.54 \\
\hline \multirow{3}{*}{$\begin{array}{l}\text { Probabilisti } \\
\text { c neural } \\
\text { networks }\end{array}$} & Zone 1,2 and 3 & 8.27 & 8.2 \\
\hline & Zone $2,3,4,6,8 \& 9$ & 14.7 & 10.54 \\
\hline & Entire image & 19.7 & 23.5 \\
\hline \multirow{3}{*}{$\begin{array}{l}\text { Support } \\
\text { vector } \\
\text { machines }\end{array}$} & Zone 1,2 and 3 & 12.23 & 9.7 \\
\hline & Zone $2,3,4,6,8 \& 9$ & 25.5 & 34.7 \\
\hline & Entire image & 21.22 & 20.46 \\
\hline
\end{tabular}

\begin{tabular}{|c|c|c|c|c|}
\hline Classifier & Zone selection & $\begin{array}{l}\text { Prec } \\
\text { isio } \\
\mathbf{n} \\
\% \\
\end{array}$ & $\begin{array}{l}\text { F- } \\
\text { Meas } \\
\text { ure } \\
\% \\
\end{array}$ & $\begin{array}{l}\text { Acc } \\
\text { urac } \\
\text { y } \\
\% \\
\end{array}$ \\
\hline \multirow{3}{*}{$\begin{array}{c}\text { Nearest } \\
\text { neighbor }\end{array}$} & Zone 1,2 and 3 & 0.11 & 0.2 & 3.2 \\
\hline & Zone $2,3,4,6,8 \& 9$ & 0.4 & 0.77 & 15.7 \\
\hline & Entire image & 0.3 & 0.57 & 4.71 \\
\hline \multirow{3}{*}{$\begin{array}{l}\text { Naïve } \\
\text { Bayes }\end{array}$} & Zone 1,2 and 3 & 0.23 & 0.44 & 3.81 \\
\hline & Zone $2,3,4,6,8 \& 9$ & 0.9 & 1.72 & 18.9 \\
\hline & Entire image & 0.46 & 0.88 & 7.7 \\
\hline \multirow{3}{*}{$\begin{array}{l}\text { Probabilist } \\
\text { ic neural } \\
\text { networks }\end{array}$} & Zone 1,2 and 3 & 0.31 & 0.59 & 8.2 \\
\hline & Zone $2,3,4,6,8 \& 9$ & 0.57 & 1.09 & $\begin{array}{r}10.6 \\
8 \\
\end{array}$ \\
\hline & Entire image & 0.9 & 1.72 & 23.3 \\
\hline
\end{tabular}




\begin{tabular}{|c|l|r|r|r|} 
& Zone 1, 2 and 3 & 0.47 & 0.9 & 9.79 \\
\cline { 2 - 5 } $\begin{array}{c}\text { Support } \\
\text { vector } \\
\text { machines }\end{array}$ & Zone 2,3,4,6,8 \& 9 & 1.37 & 2.59 & 34.4 \\
\cline { 2 - 5 } & Entire image & 0.92 & 1.76 & $\begin{array}{r}20.4 \\
8\end{array}$ \\
\hline
\end{tabular}

Table 4. Experimental evaluation- Performance of classifiers- Hu Moments features

\begin{tabular}{|c|c|c|c|}
\hline Classifier & Zone selection & $\begin{array}{l}\text { Sensi } \\
\text { tivity } \\
\% \\
\end{array}$ & $\begin{array}{l}\text { Specif } \\
\text { icity } \\
\%\end{array}$ \\
\hline \multirow{3}{*}{$\begin{array}{c}\text { Nearest } \\
\text { neighbor }\end{array}$} & Zone 1,2 and 3 & 2.51 & 4.36 \\
\hline & Zone $2,3,4,6,8 \& 9$ & 18.7 & 14.9 \\
\hline & Entire image & 9.35 & 12.43 \\
\hline \multirow[b]{3}{*}{ Naïve Bayes } & Zone 1,2 and 3 & 6.83 & 11.03 \\
\hline & Zone $2,3,4,6,8 \& 9$ & 40.2 & 34.5 \\
\hline & Entire image & 16.9 & 13.62 \\
\hline \multirow{3}{*}{$\begin{array}{c}\text { Probailistic } \\
\text { neural } \\
\text { networks }\end{array}$} & Zone 1,2 and 3 & 8.27 & 9.9 \\
\hline & Zone $2,3,4,6,8 \& 9$ & 34.1 & 25.05 \\
\hline & Entire image & 21.94 & 27.96 \\
\hline \multirow{3}{*}{$\begin{array}{l}\text { Support } \\
\text { vector } \\
\text { machines }\end{array}$} & Zone 1,2 and 3 & 16.18 & 9.9 \\
\hline & Zone $2,3,4,6,8 \& 9$ & 38.1 & 43.8 \\
\hline & Entire image & 32.01 & 36.75 \\
\hline
\end{tabular}

\begin{tabular}{|c|c|c|c|c|}
\hline Classifier & Zone selection & $\begin{array}{l}\text { Precis } \\
\text { ion } \\
\%\end{array}$ & $\begin{array}{l}\text { F- } \\
\text { Mea } \\
\text { sure } \\
\%\end{array}$ & $\begin{array}{l}\text { Accur } \\
\text { acy } \\
\%\end{array}$ \\
\hline \multirow{3}{*}{$\begin{array}{c}\text { Nearest } \\
\text { neighbor }\end{array}$} & Zone 1,2 and 3 & 0.09 & 0.17 & 4.3 \\
\hline & Zone $2,3,4,6,8 \& 9$ & 0.76 & 1.46 & 15.1 \\
\hline & Entire image & 0.37 & 0.71 & 12.32 \\
\hline \multirow{3}{*}{$\begin{array}{l}\text { Naïve } \\
\text { Bayes }\end{array}$} & Zone 1,2 and 3 & 0.26 & 0.50 & 10.8 \\
\hline & Zone $2,3,4,6,8 \& 9$ & 2.11 & 4.00 & 34.7 \\
\hline & Entire image & 0.68 & 1.30 & 13.73 \\
\hline \multirow{3}{*}{$\begin{array}{l}\text { Probailistic } \\
\text { neural } \\
\text { networks }\end{array}$} & Zone 1,2 and 3 & 0.32 & 0.61 & 9.85 \\
\hline & Zone $2,3,4,6,8 \& 9$ & 1.57 & 3.00 & 25.3 \\
\hline & Entire image & 1.05 & 2.00 & 27.75 \\
\hline \multirow{3}{*}{$\begin{array}{l}\text { Support } \\
\text { vector } \\
\text { machines }\end{array}$} & Zone 1,2 and 3 & 0.6 & 1.15 & 14.79 \\
\hline & Zone $2,3,4,6,8 \& 9$ & 2.32 & 4.37 & 43.62 \\
\hline & Entire image & 1.74 & 3.30 & 36.59 \\
\hline
\end{tabular}

The accuracy of SVM classifiers obtained from various zone selections and feature extraction methods are projected in the figure 21 for comparison.

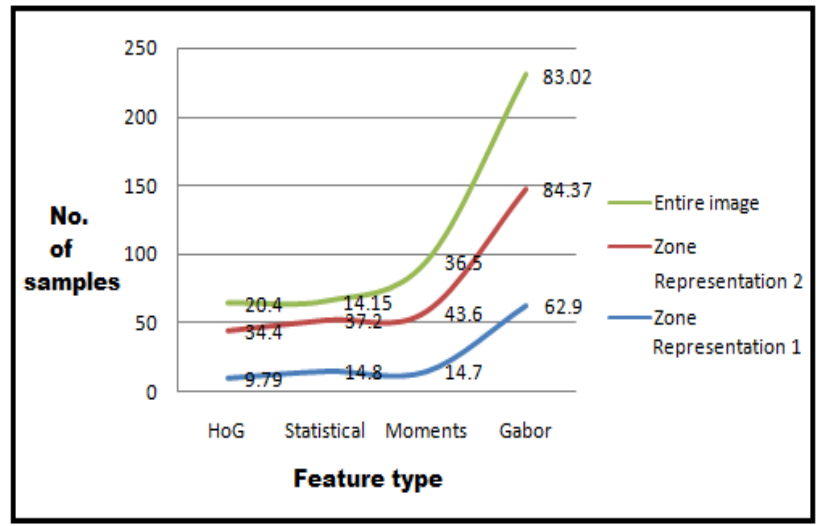

Fig. 18 Comparison of accuracy of SVM vs. Feature extraction techniques

From the experimentation, it is evident that the zone representation 2 is almost equally efficient with the character recognition using entire image. The zone representation 2 has been selected in the proposed technique in the perspective of increasing the localization of features from one character image to other. From the experimentation it is deduced that, the classifiers multi-class SVM and Probabilistic neural networks are efficient compared to Naïve Bayesian and nearest neighbor classification. Also, the feature extraction techniques employed other than Gabor features are not robust enough for recognition of handwritten Telugu characters.

\section{CONCLUSION}

Efficiency of any character recognition system mainly depends upon the feature extraction and classification techniques. In the perspective of handwritten character recognition, we have employed Gabor features extracted from two different zone representation and entire image. The discrimination of features has been performed using nearest neighbor classifier, multi-class SVM, Naïve Bayesian and probabilistic neural networks. It is observed that most of the misclassifications are happened due to increase in number of confusing character classes present in single level vowelconsonant clusters. In order to improve the recognition rate, a method is under investigation with the support of mapper module during classification process that resolves the conflicts of confusing character classes.

\section{REFERENCES}

[1] L. Heutte, T. Paquet, J.V. Moreau, Y. Leocourtier, C. Olivier, "A structural/statistical feature based vector for handwritten character recognition”, Pattern Recognition letters, Vol. 19, issue 7, pp. 629-641, 1998.

[2] Seong-Whan Lee, "Direct extraction of topographic features for gray scale character recognition", IEEE transactions on pattern analysis and machine intelligence, Vol. 17, Issue 7, pp. 724-728, 1995.

[3] Chao kan, Mandyam D. Srinath, "Invariant character recognition with Zernike and orthogonal Fourier-Mellin moments", Pattern recognition, Vol. 35, Issue 1, pp. 143$154,2002$.

[4] L. Wang, " Direct gray scale extraction of features for character recognition", IEEE transactions on pattern analysis and machine intelligence, Vol. 15, Issue 10, pp. 1053-1067, 2002 
[5] Anil K. Jain, Torfinn Taxt, " Feature extraction methods for character recognition- A Survey", Pattern Recognition, Vol. 29, Issue 4, pp. 641-662, 1996.

[6] Rajashekararadhya, S.V. Ranjan, P. vanaja, " Efficient zone-bsed feature extraction algorithm for handwritten numeral recognition of four popular South Indian scripts", Journal of theoretical and information technology, Vol. 4, Issue 12, pp. 1171-1181, 2008.

[7] John T. Favata, Geetha Srikantan, "A multiple feature/Resolution approach to hand printed digit and character recognition", International journal of imaging systems and technology, Vol. 7, pp. 304-311, 1996.

[8] George S, Kapogiannopoulos, "Character recognition using a bi-orthogonal discrete wavelet transform", Proc. SPIE, Wavelet Applications in Signal and Image Processing IV, Vol. 2825, 1996.

[9] Pritpal Singh, Sumit Budhiraja, "Feature extraction and classification techniques in OCR systems for Handwritten Gurumukhi script-A survey", International journal of engineering, research and applications, Vol. 1, Issue 4, pp. 1736-1739, 2011.

[10] Sumedha B. Hallale, Geeta D. Salunke, "Twelve directional feature extraction for handwritten English character recognition", International Journal of Recent Technology and Engineering (IJRTE), Volume-2, Issue2, pp. 39-42, 2013.

[11] Gita Sinha, Rajneesh Rani and Renu Dhir. "Handwritten Gurmukhi numeral recognition using zone-based hybrid feature extraction techniques". International Journal of Computer Applications 47(21):24-29, June 2012.

[12] [12] Sandeep Saha, Nabarag Paul, Sayam Kumar Das, sandip Kundu, "Optical Character Recognition using 40point feature extraction and artificial neural network" International Journal of Advanced Research in Computer Science and Software Engineering”, Volume 3, Issue 4, pp. 495-502, April 2013.

[13] Raju Dara, Urmila Panduga, "Telugu Handwritten Isolated Characters Recognition using Two Dimensional Fast Fourier Transform and Support Vector Machine", International Journal of Computer Applications, Vol. 116, No. 5,pp. 7-11, 2015.

[14] C. Vikram, C. Shoba Bindu, C. Sasikala, "Handwritten Character Recognition for Telugu Scripts Using Multi Layer Perceptrons (MLP)", International Journal of Advanced Research in Computer Engineering \& Technology (IJARCET), Vol. 2, No. 12, pp. 3105-3110, 2013.

[15] Ram Mohan Rao, B.Ramesh, G.Indrasena Reddy, "Font and Size Identification in Telugu Printed Document", International Journal of Engineering Research and Development, Vol. 6, No. 11. 2013, PP. 92-110.

[16] K. Vijay Kumar R.Rajeshwara Rao, "Improvement in Efficiency of Recognition of Handwritten Telugu script", International Journal of Inventive Engineering and Sciences (IJIES), Vol.-2, No. 1, pp. 1-4, 2013.

[17] Samit Kumar Pradhan, "A syntactic PR approach to Telugu handwritten character recognition", Proceeding of the workshop on Document Analysis and Recognition, pp. 147-153, ACM, 2012.
[18] Dan E. Willard,'New trie data structures which supports very fast search operations", Journal of computer and system sciences, Vol. 28, issue 3, pp.379-394, 1984.

[19] B.V. Dhandra, R.G. Benne, Mallikarjun Hangarge, "Kannada, Telugu and Devanagari Handwritten Numeral recognition with Probabilistic Neural Network: A Script Independent Approach", International

[20] Panyam Narahari Sastry, Ramakrishnan Krishnan, Bhagavatula Venkata Sanker Ram, "Classification and identification of telugu handwritten characters extracted from palm leaves using decision tree approach", ARPN Journal of Engineering and Applied Sciences ,Vol. 5, No. 3, pp. 22-32, 2010.

[21] Andrés, Javier de., "Statistical techniques vs. SEES algorithm: an application to a small business environment", 2001

[22] Venkat Rasagna, K. J. Jinesh, C. V. Jawahar, "On Multifont Character Classification in Telugu", International Institute of Information Technology, Hyderabad, INDIA.

[23] Nusaibath C, Ameera Mol P M, "Off-line Handwritten Malayalam Character Recognition Using Gabor Filters", International Journal of Computer Trends and Technology (IJCTT), Vol. 4 No. 8, pp. 2476-2479, 2013.

[24] Xuewen Wang, Xiaoqing Ding, Changsong Liu, "Gabor filters-based feature extraction for character recognition", Pattern Recognition, Vol. 38, No. 3, pp. 369-379, 2005.

[25] C. L. Liu ; Central Res. Lab., Hitachi Ltd., Kokubunji, Japan ; M. Koga ; H. Fujisawa, "Gabor feature extraction for character recognition: comparison with gradient feature", International conference on Document Analysis and Recognition, Vol. 1, pp. 121 - 125, 2005.

[26] Xuewen Wang, Xiaoqing Ding, Changsong Liu, "Optimized Gabor filter based feature extraction for character recognition", International Conference on Pattern Recognition, IEEE, Vol. 4, pp. 223-226, 2002.

[27] Jin Chen, Huaigu Cao, Rohit Prasad, Anurag Bhardwaj, Prem Natarajan, "Gabor Features for Offline Arabic Handwriting Recognition", Workshop on Document Analysis and Systems, 2010.

[28] Liu, C., Wechsler, H., "Independent component analysis of Gabor features for face recognition", IEEE transactions on Neural Networks, Vol. 14, issue 4, pp.919-928, 2003.

[29] Chacko, B.P., Krishnan, V.V., Raju, G., Anto, P.B., "Handwritten character recognition using wavelet energy and extreme learning machine", International Journal of Machine Learning and Cybernetics, Vol. 3, issue 2, pp.149-161, 2012.

[30] Cash, G.L., Hatamian, M., "Optical character recognition by the method of moments", Computer Vision, Graphics, and Image Processing, Vol. 39, issue 3, pp.291-310, 1987.

[31] LeCun, Y., Bottou, L., Bengio, Y., Haffner, P., "Gradient-based learning applied to document recognition”, Proceedings of the IEEE, Vol. 86, issue 11, pp.2278-2324, 1998. 
[32] Tan, Hin-Leong., "Hybrid feature-based and template matching optical character recognition system", U.S. Patent No. 5,077,805, 31 Dec. 1991.

[33] P.B. Pati, S. Sabari Raju, N. Pati, A. G. Ramakrishnan, "Gabor filters for document analysis in Indian bilingual documents", International Conference on Intelligent Sensing and Information Processing, pp. 123-126, 2004.

[34] Jonathan Alon, Vassilis Athitsos, and Stan Sclaroff, "Online and Offline Character Recognition Using Alignment to Prototypes", International Conference on Document Analysis and Recognition (ICDAR), 2005.

[35] Chih-Wei Hsu, Chih-Jen Lin," A Comparison of Methods for Multi-class Support Vector Machines", IEEE Transactions on Neural Networks, Vol.13(2), pp. 415-425, 2002.

[36] Sabine Barrat, Salvatore Tabbone, Patrick Nourrissier ,A Bayesian classifier for symbol recognition", Workshop on Graphics Recognition - GREC, 2007.
[37] Richard D. Romero, David S. Touretzky, Robert H. Thibadeau, "Optical Chinese character recognition using probabilistic neural networks", Pattern Recognition, Vol. 30, Issue 8, pp. 1279-1292, 1997.

[38] Vincent Labatut, Hocine Cherifi, "Accuracy measures for comparision of classifiers", International conference on information technology, arXiv:1207.3790, 2011.

[39] Newell, Andrew J., Lewis D. Griffin, "Multiscale histogram of oriented gradient descriptors for robust character recognition", In 2011 International Conference on Document Analysis and Recognition, pp. 1085-1089, IEEE, 2011

[40] Davis, James W., Aaron F. Bobick, "The representation and recognition of human movement using temporal templates", In Computer Vision and Pattern Recognition, 1997. Proceedings., 1997 IEEE Computer Society Conference on, pp. 928-934, IEEE, 1997. 